\title{
Investigation of influence factors on residual stress determination within coated surfaces in consideration of the differential and integral method
}

\author{
Peter Weidmann ${ }^{1, a^{*}}$, Ulrich Weber ${ }^{1, b}$, Siegfried Schmauder ${ }^{1, c}$ \\ and Venancio Martínez García ${ }^{2, d}$ \\ ${ }^{1}$ IMWF, University of Stuttgart, Pfaffenwaldring 32, 70569 Stuttgart, Germany \\ ${ }^{2}$ IFKB, University of Stuttgart, Allmandring 7b, 70569 Stuttgart, Germany \\ apeter.weidmann@imwf.uni-stuttgart.de, bulrich.weber@imwf.uni-stuttgart.de, \\ csiegfried.schmauder@imwf.uni-stuttgart.de, ${ }^{d}$ venancio.martinez@gsame.uni-stuttgart.de
}

Keywords: Residual stress, differential method, integral method, coated materials.

\begin{abstract}
To improve the surface behavior of metal structures, like wear- and heat resistance or hardness, often thermally sprayed ceramic coatings are applied. A modern technology to realize dense layers is the High Velocity Oxygen Fuel (HVOF) technique. The deposition process and necessary pretreatments can cause high residual stresses within the coating and the substrate. While tensile stresses in the brittle coating may cause cracks, compressive stress states can even improve the materials behavior. To guarantee high product quality, it is necessary to know exactly the occurring residual stresses in the metal-ceramic hybrid system. A very common measurement technique is the incremental hole drilling (IHD) method. To determine the residual stresses out of IHD measurements the differential method (DM) or integral method (IM) can be used. To investigate the influence of interfacial layers, nonlinear stresses, cracking, anisotropy, variation of coating thickness and calculation formalisms several FEM models have been build, while case sensitive calibration was used for the coated systems.
\end{abstract}

\section{Introduction}

The IHD method is a common technique to determine residual stresses, where a stepwise hole is drilled into residual stress afflicted material. The hereby occurring surface strain can be measured with strain gauge rosettes. The two most common calculations to convert these strains into the depth-dependent stress are the differential and the integral method. Both methods rely on different assumptions and have originally been established for homogeneous materials. But within a coated system the elastic properties are not homogeneous and must be considered in case sensitive calibrations [1,2]. In order to establish a comparative study of both methods, separate FEM models have to be considered and diverse parameter studies arranged. On this basis the most appropriate method to cover the requirements and difficulties may be chosen.

\section{Theoretical basics of the differential and the integral method}

The aim of both methods is to transfer the measured surface strains $\varepsilon$ in related residual stresses per depth increment $h$. The DM is based on the assumption that the surface strain change $d \varepsilon / d h$ is related to nominal strains $\varepsilon_{\mathrm{n}}$ within the corresponding depth increment [3]. The relation between the surface strain change and the nominal strain is given by the calibration function $\mathrm{K}$. Taking into account the X-direction on the surface and assuming the residual stresses $\sigma_{\mathrm{x}}$ and $\sigma_{\mathrm{y}}$ the surface strain change can be calculated with Eq. 1 . Hereby $\varepsilon_{n x y}$ is the in depth nominal strain in the Xdirection which arises from the stress in the Y-direction. For isotropic materials the Poissons Ratio $\mu$, where $\varepsilon_{\mathrm{nx}}=-\mu \varepsilon_{\mathrm{ny}}$, can be introduced. With Eq. 1 for both directions this leads to Eq. 2

$$
\frac{d \varepsilon_{x}(h)}{d h}=K_{x}(h) \cdot \varepsilon_{n x x}(h)+K_{y}(h) \cdot \varepsilon_{n x y}(h) .
$$




$$
\varepsilon_{n x x}(h)=\frac{1}{K_{x}^{2}(h)-\mu^{2} \cdot K_{y}^{2}(h)}\left[K_{x}(h) \frac{d \varepsilon_{x}(h)}{d h}+\mu \cdot K_{y}(h) \frac{d \varepsilon_{y}(h)}{d h}\right] .
$$

To equate the residual stresses Hookes' law can now be used. As the elastic properties of coating and substrate are different the elastic modulus and the Poissons Ratio must be related to the particular depth $\mathrm{h}[1]$. This leads to

$$
\sigma_{x}(h)=\frac{E(h)}{K_{x}^{2}(h)-\mu^{2}(h) \cdot K_{y}^{2}(h)}\left[K_{x}(h) \frac{d \varepsilon_{x}(h)}{d h}+\mu(h) \cdot K_{y}(h) \frac{d \varepsilon_{y}(h)}{d h}\right] .
$$

After transposing the last two equations the calibration functions may be determined if a known stress state is given. For every combination of material and coating thickness separate calibration functions $\mathrm{K}$ have to be calculated.

With the given equations it can be seen that the DM assumes that only residual stresses within the cutting increment are responsible for the surface deformation. Another technique which transposes the residual stress of every depth in a corresponding surface strain is the IM [4]. Within this method the whole stress-state is expressed in its hydrostatic $\mathrm{P}(\mathrm{h})$ and deviatoric parts. Additionally, the IM takes into account the stress values of previous depths. The current cutting depth is still expressed as $h$ while the depth of the considered residual stress is $H$. The IM then assumes that the relaxed surface strain, i.e. $\mathrm{p}(\mathrm{h})$, is the integral of the infinitesimal strain components which occur due to the stresses $\mathrm{P}(\mathrm{h})$ from the depths $\mathrm{h}$ (Eq. 4). For coated surfaces the elastic properties $\mathrm{E}$ and $\mu$ depend on the depth $\mathrm{h}$ and are, therefore, no longer scalars. Hereby $\hat{\mathrm{A}}(\mathrm{H}, \mathrm{h})$ describes the relation between the in plane stress and its corresponding surface strain $\mathrm{p}(\mathrm{h})$. Transformation into a discrete form with $\mathrm{n}$ increments leads to Eq. 5.

The index $\mathrm{i}$ accounts for the hole depth and $\mathrm{j}$ for the depth increment. The factor $\mathrm{a}_{\mathrm{ij}}$ relates to $\hat{\mathrm{A}}(\mathrm{H}, \mathrm{h})$ and, therefore, describes the amount of surface strain relaxation due to the increment $\mathrm{j}$ at a hole $\mathrm{i}$ increments deep. In matrix notation, Eq. 5 may be written as Eq. 6. Due to the nonhomogenous material behavior the elastic properties $\mathrm{E}$ and $\mu$ are hereby vectors. Now the matrix $\overline{\mathrm{a}}$ contains coefficients related to hole deformations when cutting a hole through a hydrostatic stress field and an additional matrix $\bar{b}$ related to hole deformations when cutting through a pure shear field. Both matrices are always lower triangular and must be calculated separately for every combination of materials and coating thicknesses [2]. If the calibration constants are known, the residual stresses can be determined with the measured surface strains.

$$
\mathrm{p}(\mathrm{h})=\frac{1+\mu(\mathrm{h})}{\mathrm{E}(\mathrm{h})} \int_{0}^{\mathrm{h}} \hat{\mathrm{A}}(\mathrm{H}, \mathrm{h}) \cdot \mathrm{P}(\mathrm{h}) \mathrm{dH} \rightarrow \sum_{\mathrm{i}, \mathrm{j}=1}^{\mathrm{i}, \mathrm{j}=\mathrm{n}} \overline{\mathrm{a}}_{\mathrm{ij}} \mathrm{P}_{\mathrm{i}}=\frac{\mathrm{E}_{\mathrm{i}}}{1+\mu_{\mathrm{i}}} \mathrm{p}_{\mathrm{i}} \rightarrow \overline{\mathrm{a}} \mathrm{P}=\frac{\mathrm{E}}{1+\mu} \mathrm{p} .
$$

\section{Numerical models and experiments}

As shown with above equations, calibration functions and coefficients can be determined if the surface deformation due to the IHD process for a well-known stress-state is measured. Therefore, Finite Element simulations are suitable. In this work, separate $3 \mathrm{~d}$ models for the DM and the IM have been built with the software ABAQUS 6.12-1. Because of the symmetrical conditions they consist of a quarter of the cutting hole. The cutting hole diameter $\mathrm{D}_{\mathrm{o}}$ was chosen to be $1.8 \mathrm{~mm}$. The modeled strain gauges have the size of Type B - 1/16 inch nominal [4]. Regarding the integrative effect of the strain gauges the average strain of the strain gauge area was calculated. Both models are similar in geometry and mesh (Fig. 1, Fig. 2). For the DM two strain gauges must be modeled while one is sufficient for the IM. The maximum hole depth was $1 \mathrm{~mm}$ with $0.05 \mathrm{~mm}$ steps for the IM model. The residual stress was usually applied as a predefined stress field in equilibrium to the outer boundaries. Only for the IM coefficients the stress distribution was simulated directly on the cutting hole surface. The cutting process was realized through the deletion of the appropriate elements per cutting-increment. The IM model consisted of $15680 \mathrm{C} 3 \mathrm{D} 20$ elements. The elastic properties were $\mathrm{E}_{\mathrm{s}}=210000 \mathrm{MPa}$ and $\mu_{\mathrm{s}}=0.27$ for the steel substrate and $\mathrm{E}_{\mathrm{c}}=165850 \mathrm{MPa}$ and 
$\mu_{\mathrm{c}}=0.23$ for the coating, which simulates an alumina coating. In order to determine the calibration coefficients $\mathrm{K}$, the strain-curves were approximated with polynomials of $6^{\text {th }}$ order. Also the coefficient functions could be expressed as polynomials but due to the different elastic properties different polynomials had to be used for the coating and the substrate.

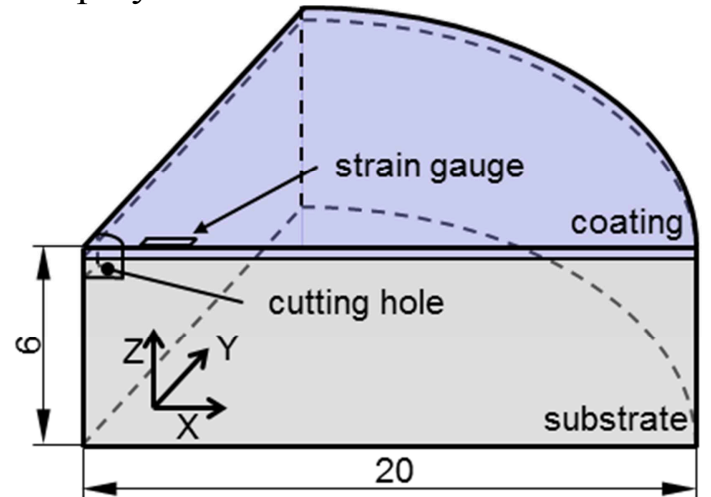

Fig. 1: Geometric properties of the IM FE model

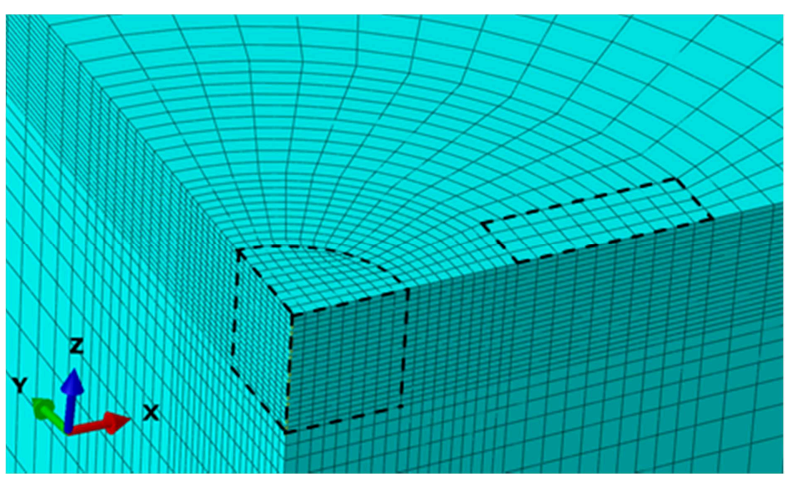

Fig. 2: Mesh of the IM model with marked cutting hole and strain gauge

With the introduced model the influence of coating thickness was investigated with models of different alumina heights $(0,20,80$ and $120 \mu \mathrm{m})$. Also two inhomogeneous through thickness residual stresses have been numerically analyzed. One with $100 \mathrm{MPa}$ in the coating and $-50 \mathrm{MPa}$ in the substrate and one with a linear distribution of 0 to $100 \mathrm{MPa}$ from the surface to a depth of $1 \mathrm{~mm}$. In both cases the appropriate residual stress was applied separately on every cutting increment layer. As the coating process is done in layers its behavior may be anisotropic. Therefore, additional models with orthotropic coatings have been built. Their transversal elastic properties $\left(E_{t}=210000 \mathrm{MPa}\right)$ were constant while the one in the lateral direction differed $\left(E_{t} / E_{1}=0.8\right.$ and $\mathrm{E}_{\mathrm{t}} / \mathrm{E}_{1}=0.6$ ) for a coating thickness of 100 and $200 \mu \mathrm{m}$. Some other preliminary investigations showed that the results are completely independent of the in plane residual stress state, therefore this parameter wasn't examined further.

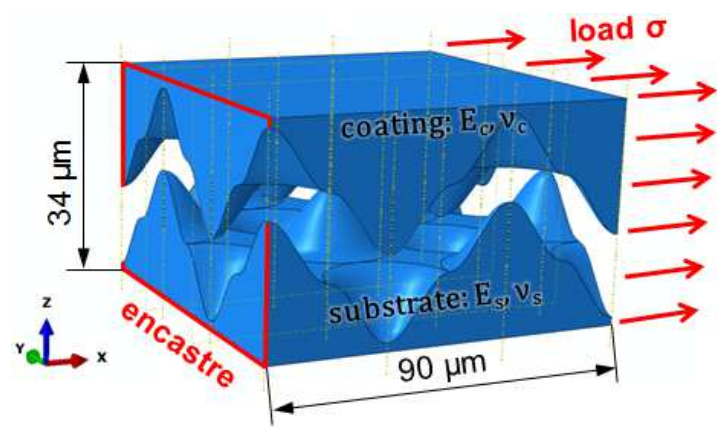

To investigate the influence of the rough interface between substrate and coating a separate model was build. It consists of two parts with sinusoidal ex- and intruded parts to approximate the sand blasted surface (Fig. 3). The elastic properties are the same as mentioned before for a steel substrate with alumina coating. Between both parts a tie constraint was applied. Additionally this constraint was reduced to simulate a non-optimal bonding. If a pressure load is

Fig. 3: Rough interface regime with boundary conditions (exploded view) applied on one surface of the whole model the length change can be measured and new global elastic properties $\mathrm{E}_{\mathrm{i}}=\sigma \mathrm{l}_{0} / \Delta \mathrm{l}$ and $\mu_{\mathrm{i}}=-\mathrm{1}_{0} / \Delta \mathrm{l} \cdot \Delta \mathrm{d} / \mathrm{d}_{0}$ may be calculated. Overall this model consists of $713811 \mathrm{C} 3 \mathrm{D} 10$ tetrahedral elements. With its dimensions it refers to a roughness of $\mathrm{R}_{\mathrm{Z}}=30 \mu \mathrm{m}$, measured on the substrate after sandblasting. The calculated elastic properties of this model could then be applied on a quadratic layer between coating and substrate in the model shown in Fig. 1.

Ceramic coatings like alumina bear a brittle behavior, especially if tensile residual stresses appear [5]. To investigate the effects of brittle damage during IHDs on the measured surface strains and the form of fracture appearance it was necessary to establish an own model. Therefore a 2D model of the half cross section with a coating thickness of $0.2 \mathrm{~mm}$ was build (Fig. 4). It consisted of 5532 CPE4R elements. The coating material behavior was modeled with Abaqus' Brittle Cracking option. Hereby fracture initiates at an initiation stress $\sigma_{\text {ini }}$ while the crack propagation is calculated with the materials fracture energy $G_{f}$ and a retention factor $\rho_{\mathrm{r}}$. The retention factor characterizes the degeneration of the shear modulus due to a crack-dependent strain $\varepsilon$. After a critical crack opening 
displacement $\mathrm{u}_{\mathrm{f}}$ has been hit the correspondent elements are deleted. Preliminary numerical investigation showed that a linear retention factor distribution between the points $\left(\varepsilon, \rho_{\mathrm{r}}\right)=(0,1) ;(0.0001,0.01) ;(0.001,0) \quad$ with $\quad \mathrm{u}_{\mathrm{f}}=3 \mathrm{e}-5 \quad$ is appropriate. $\sigma_{\text {ini }}$ was chosen to be $100 \mathrm{MPa}$ with $\mathrm{G}_{\mathrm{f}}=0.02 \mathrm{~N} / \mathrm{mm}$.

To achieve a better resolution for residual stress determinations out of real strain measurements another IM model was built with step increments of $20 \mu \mathrm{m}$ from 0 to 0.5 $\mathrm{mm}$ depth and $50 \mu \mathrm{m}$ afterwards to the maximum depth of $0.8 \mathrm{~mm}$. With the calculated calibration functions and

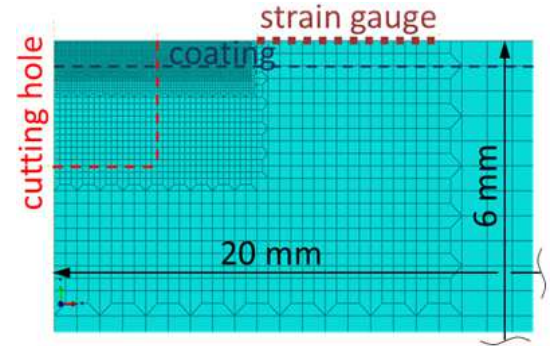

Fig. 4: Mesh detail of the brittle cracking model coefficients residual stress determinations with IHD steps of $10 \mu \mathrm{m}$ were performed on HVOF sprayed coatings with a thickness of $100 \mu \mathrm{m}$ on steel- and $200 \mu \mathrm{m}$ on aluminum-substrate. The substrate surfaces were pretreated by sandblasting. For the DM the strain was approximated sequentially with polynomials of second degree. Therefore, an area of $\mathrm{m}$ points around the strainmeasurement $\varepsilon_{\mathrm{i}}(\mathrm{h})$ is defined. This area can be approximated with a polynomial $\mathrm{P}$ (least-squares method). So the smoothening depends on $\mathrm{m}$ while the derivative $\mathrm{P}^{\prime}$ can easily be calculated. The smoothening is always chosen to be within the measurements root mean square error. To acquire smooth curves with the IM a Tikhonov regularization is practicable [4]. Hereby Eq. 6 is extended with the matrix $\overline{\mathrm{c}}$, which consists of $-1,2,-1$ on the diagonal and zero in the first and last line (Eq. 7). But this equation cannot be applied on non-isotropic materials, as the elastic properties have to be applied as a vector. Therefore, Eq. 7 would lead to a wrong weighting of the coefficients. It is proposed here to use a calibration matrix with integrated elastic properties $\bar{a}_{\mathrm{ei}}$ in the form of $\overline{\mathrm{a}}_{\mathrm{ei}}(\mathrm{i}, \mathrm{j})=\overline{\mathrm{a}}\left(1+\mu_{\mathrm{j}}\right) / \mathrm{E}_{\mathrm{j}}$. This leads to the adapted Tikhonov equation (8).

$$
\left(\overline{\mathrm{a}}^{\mathrm{T}} \overline{\mathrm{a}}+\alpha_{\mathrm{P}} \overline{\mathrm{c}}^{\mathrm{T}} \overline{\mathrm{c}}\right) \mathrm{P}=\frac{\mathrm{E}(\mathrm{h})}{1+\mu(\mathrm{h})} \overline{\mathrm{a}}^{\mathrm{T}} \mathrm{p} \rightarrow\left(\overline{\mathrm{a}}_{\mathrm{ei}}^{\mathrm{T}} \overline{\mathrm{a}}_{\mathrm{ei}}+\alpha_{\mathrm{P}} \overline{\mathrm{c}}^{\mathrm{T}} \overline{\mathrm{c}}\right) \mathrm{P}=\overline{\mathrm{a}}_{\mathrm{ei}}^{\mathrm{T}} \mathrm{p}
$$

\section{Results}

If residual stresses for the DM are calculated without case sensitive calibration functions the simulations showed mistakes up to $24 \%$ (for a coating of $0.2 \mathrm{~mm}$ ). If case sensitive calibration is done within an accuracy of $\pm 50 \mu \mathrm{m}$ for a $100 \mu \mathrm{m}$ coating the mistake lies mostly under $3 \%$. Roughly the same could be seen for IM determinations. For all numerical investigations no regression or regularization was used.

The interface simulation showed that the combined elastic properties $\mathrm{E}_{\mathrm{i}}$ of coating and substrate lead to a collective elastic modulus of $E_{i}=\left(E_{s}+E_{c}\right) / 2$. The same accounts for the Poissons Ratio and is even valid for a reduced bonding, where the tie constraint was only applied on $50 \%$ of the interface. This behavior could then easily be assigned on a model with an interface layer of $40 \mu \mathrm{m}$ and a coating thickness of $80 \mu \mathrm{m}$. Then an IHD measurement was simulated. For the DM this interface leads to a mistake of less than $1 \%$ on the surface and less than $2 \%$ within the maximum cutting depth. For the IM a mistake of less than 2\% occurred within the coating. For both calculations, case sensitive calibrations for a coating thickness of $100 \mu \mathrm{m}$ were used.

For inhomogeneous stresses the DM showed a mistake of up to $60 \%$ (Fig. 5). Especially for fast changing stress states the calculation ( $\sigma_{-}$II) converges slowly towards the real value ( $\sigma_{-}$II ref.). The IM was able to match the applied stress without any diverges. This is an already known behavior for homogenous materials [6].

A transversal isotropic coating behavior leads to mistakes in residual stress determinations of up to $40 \%$ for DM and $20 \%$ for IM calculations (Fig. 6). Hereby the quotient $E_{t} / E_{1}$ has only little influence compared to the impact of the coating thickness. I.e. within a $100 \mu \mathrm{m}$ coating the mistake for the $\mathrm{DM}$ is under $10 \%$ for $\mathrm{E}_{\mathrm{t}} / \mathrm{E}_{1}=0.6$ on the top surface and $5 \%$ for the IM. The errors due to transversal isotropy can be reduced if the calibration functions and coefficients are calculated with 
appropriate anisotropic FEM models. For these case sensitive calibrations the deviation for the IM could be lowered to a maximum of 5\% and 3\% for the DM (Fig. 6).

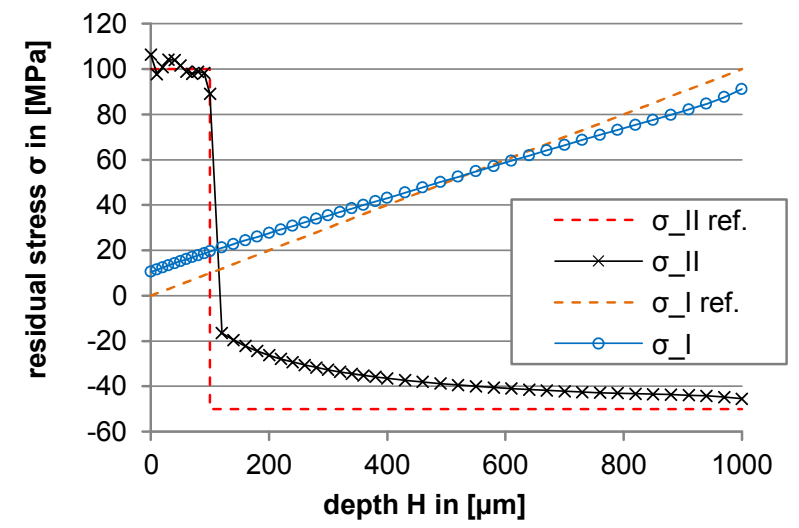

Fig. 5: Inhomogeneous through thickness residual stress (I - linear distribution, II - stress leap); DM determination; FEM calculation

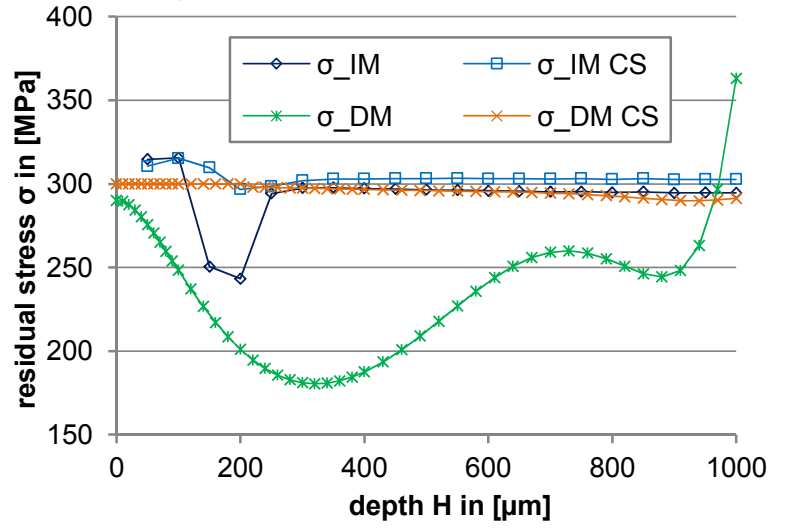

Fig. 6: DM/IM determination with and without case sensitive (CS) calibration; $0.2 \mathrm{~mm}$ transversal isotropic coating; $\mathrm{E}_{\mathrm{t}} / \mathrm{E}_{1}=0.8 ; \mathrm{FEM}$ calculation

The main impact of occurring fractures during the drilling process can be seen in the released surface strain, as a transversal crack will increase suddenly the stress of deeper increments (Fig. 7, right). The first measurable cracks appeared when the residual stress reached $60 \%$ of the failure initiation stress $\sigma_{\text {ini. }}$. The cracks always started at the edge of the cutting-hole bottom in a transversal way and stopped at the substrate (Fig. 7, left). This is important to keep in mind during IHD measurements, because strain discontinuities may arise from such crack formations. For lower critical crack opening displacements $\mathrm{u}_{\mathrm{f}}$ and higher stresses (ca. $0.8 \cdot \sigma_{\text {ini }}$ ) a complete delamination of the coating can appear (Fig. 7, middle).
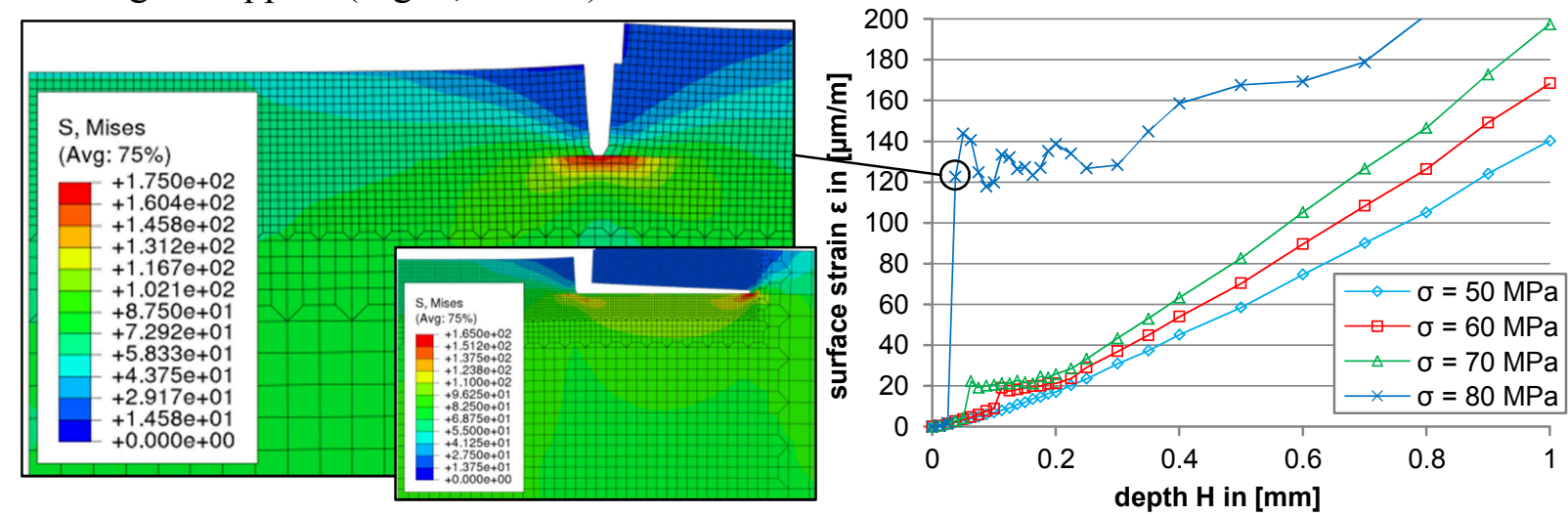

Fig. 7: Lateral fracture after a drilling depth of $30 \mu \mathrm{m}$ (left); coating delamination for lowered $\mathrm{u}_{\mathrm{f}}$ (middle); surface strains of the brittle model for different residual stresses (right)

The measurements of the HVOF sprayed coatings on steel and aluminum showed comparable residual stress trends of the DM and IM but with partly high relative differences (Fig. 8). Hereby the differential strains were approximated within a range of $\mathrm{m}=11$ points. The IM regression values laid between $10^{-10} \leq \alpha \leq 10^{-15}$. The highest stresses appeared near the interface between coating and substrate. The IM determines stresses which are up to $40 \%$ higher than the DM estimates. Also with the DM a constant stress degrading towards zero after a depth of $300 \mu \mathrm{m}$ can be seen while the IM shows clearly a tensile stress of over $60 \mathrm{MPa}$ after the depth $360 \mu \mathrm{m}$. It has to be mentioned that the maximum stress of $-935 \mathrm{MPa}$ (IM) is higher than the yield strength of the aluminum. Therefore, local plastification is likely which leads to an overestimation of residual stress determinations. This effect may be reduced due to the sandblasting induced cold working but would still be far over the materials tensile strength (max. $300 \mathrm{MPa}$ ). This may be possible because of high bearable true strain within the aluminum alloy for pressure stresses. Furthermore, the real stress state is unknown, therefore nothing can be said about the measurement deviation as well as the correctness of the DM and IM stress determination itself. 


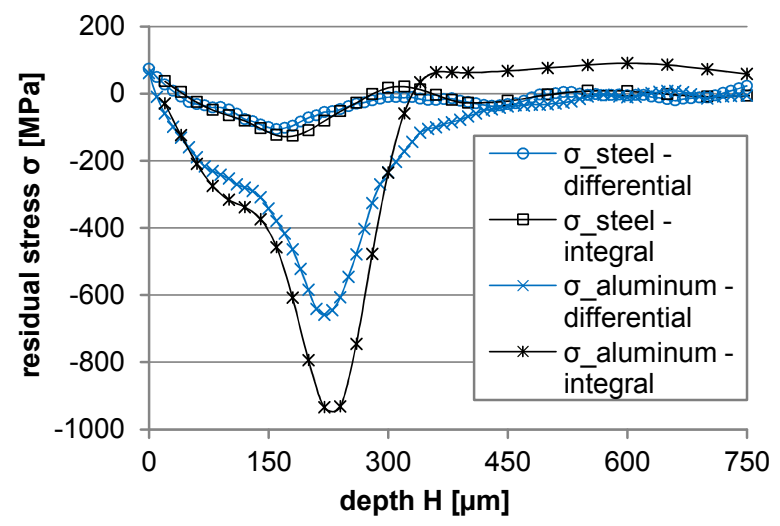

Fig. 8: DM and IM stress determination on steel- and aluminum-substrate; measurement

\section{Conclusion}

The differential as well as the integral method are, within certain limits, able to calculate the depth dependent residual stress out of IHD measurements for $\mathrm{Al}_{2} \mathrm{O}_{3}$ coated metals. Hereby the regression of strains for the DM and the adapted Tikhonov regularization seem to be suitable. The calculation of calibration functions for the DM is, compared to the IM, easier. While the DM only needs one calculation step per depth increment (i.e. 45 steps in this work) the amount of steps $\mathrm{n}_{\mathrm{s}}$ for the IM with $\mathrm{n}$ increments is $\mathrm{n}_{\mathrm{s}}=1+2+3+\ldots+\mathrm{n}$ (406 steps in this work with $\mathrm{n}=28)$. It is therefore easy to gain a high resolution for the DM and additionally the boundary conditions for the IM (i.e., depth dependent loads) are more difficult to simulate. Multi-axial inplane stress states showed no influence on the residual stress determination and errors fewer than $3 \%$ can be expected for interfacial layers of $40 \mu \mathrm{m}$. Case sensitive calibrations with an accuracy of $\pm 50 \mu \mathrm{m}$ regarding the real coating thickness must be used to guarantee a derivation within $5 \%$. Especially for high coating thicknesses the influence of transversal isotropy is up to $40 \%$ for the DM and $20 \%$ for the IM. These errors can clearly be reduced with an anisotropic case sensitive calibration. While the IM is fully capable of determine inhomogeneous through thickness stresses the DM is not able to include the residual stress of former drilling steps as it only calculates the current strain change $\mathrm{d} \varepsilon_{\mathrm{y}}(\mathrm{h}) / \mathrm{dh}$. Therefore, errors up to $60 \%$ occurred within DM determinations. As the expected residual stress within $\mathrm{Al}_{2} \mathrm{O}_{3}$ coated metals is of a highly non-homogeneous nature the IM should be preferred. A simulation of fracture mechanics within coated materials could be established. Hereby fractures in the coating always started at the bottom edge of the cutting hole if the tensile residual stress reached $60 \%$ of $\sigma_{\text {ini }}$. This may even lead to a complete delamination of the coating.

Future efforts should be done in order to experimentally validate the imposed numerical models, i.e. with the impression of predefined residual stresses. Also the coating was always modeled as a homogeneous material while in reality the HVOF process leads to a rather inhomogeneous structure [1].

\section{References}

[1] M. Wenzelburger, D. López and R. Gadow: Surface \& Coatings Technology Vol. 201 (2006), pp. 1995 - 2001

[2] T. Valente, C. Baruli, M. Sebastiani and A. Loreto: Journal of Thermal Spray Technology Vol. 14 (2005), pp. 462 - 470

[3] T. Schwarz: Beitrag zur Eigenspannungsermittlung an isotropen, anisotropen sowie inhomogen, schichtweise aufgebauten Werkstoffen mittels Bohrlochmethode und Ringkernverfahren, MPA University of Stuttgart (1996)

[4] ASTM E 837: Standard Test Method for Determining Residual Stresses by the Hole-Drilling Strain-Gage Method, ASTM International (2008)

[5] J. Malzbender, T. Wakui, E. Wessel and R. Steinbrech: Fracture Mechanics of Ceramics Vol.6 (2005), pp. $421-435$

[6] F. Haase: Eigenspannungsermittlung an dünnwandigen Bauteilen und Schichtverbunden, Shaker Verlag, Aachen (1998) 